Polymer Journal, Vol. 10, No. 1, pp 69-75 (1978)

\title{
ESR Study of the Propagating Radical in the Polymerization of MMA in Frozen State
}

\author{
Mikiharu Kamachi, ${ }^{*}$ Masahiro Kohno, ${ }^{* *}$ Der Jang Liaw, \\ and Shuhei KATSUKI* \\ *Department of Polymer Science, Faculty of Science, Osaka University, \\ Toyonaka, Osaka 560, Japan. \\ **Japan Electron Optics Laboratory Company, Akishima, Tokyo 196, Japan.
}

(Received July 8, 1977)

\begin{abstract}
An ESR study on the radical polymerization of methyl methacrylate and its homologs was carried out in frozen aromatic solvents, using benzoyl peroxide as a photosensitizer. A well-resolved 9-line spectrum was observed and its intensity distribution was found to change with temperature and the medium. In order to elucidate the origin of the 9 lines, deuterated methyl methacrylates and triphenylmethyl methacrylate having a bulky ester group were used. A 4-line spectrum $(1: 3: 3: 1)$ was observed for methyl methacrylate- $\beta-d_{2}\left(\mathrm{MMA}-\beta-d_{2}\right)$. A 3-line spectrum, whose intensity distribution was not binomial, was recorded for methyl- $d_{3}$ methacrylate (MMA- $d_{3}$ ). A 5-line spectrum $(1: 4: 6: 4: 1)$ was recorded for triphenylmethyl methacrylate. The temperature dependence of these spectra was also investigated. It was concluded from these results that the two conformations of the propagating radicals are responsible for the 9-line spectrum of methyl methacrylate in glassy aromatic solvents.

KEY WORDS ESR / Propagating Radical / Radical Polymerization /

Glassy State / Methyl Methacrylate / Methyl Methacrylate- $\beta$ - $d_{2}$ /

Methyl- $d_{3}$ Methacrylate / Triphenylmethyl Methacrylate /
\end{abstract}

It was previously ${ }^{1-3}$ reported that the elementary rate constants for the radical polymerization of methacrylate homologs and vinyl benzoate in aromatic solvents were determined and that the solvent effect on the $k_{\mathrm{p}}$ values was explainable in terms of the donor-acceptor complex of the propagating radicals with aromatic solvents. The ESR study of the polymerization of methyl methacrylate (MMA) and its homologs in the solvents was undertaken in an effort to confirm the presence of the complex. Although it is preferable to perform the ESR study under the same conditions as the polymerization, this study was carried out in a glassy medium for the following reasons: (1) The propagating radical in solution is too unstable for its stationary concentration to become high enough to be detectable by ESR even under irradiation. (2) The concentration of the complex is likely to increase at lower temperatures." (3) Anisole and benzonitrile are kept in the glassy state far below their melting points.
Although many papers ${ }^{5-30}$ have been published on the ESR study of the poly(methyl methacrylate) (PMMA) radical or the poly(methacrylic acid) (PMA) radical, both of which were prepared by solid state polymerization or degradation of the polymer, an investigation of these radicals in a glassy aromatic medium has never, to our knowledge, been reported. Thus, a comparison of the ESR spectra obtained in the aromatic solvents with those reported in other media was expected to indicate whether or not this complex formation occurred in a polymerization system.

Our polymerization systems for MMA showed the usual 9-line spectrum whose intensity distribution changed with temperature and the solvent type. Although a number of papers ${ }^{5-30}$ have been published for the elucidation of the $(5+4)$-line spectrum in the polymer matrix and the spectra have been reasonably interpreted in terms of the Gaussian distribution around the most probable conformation ${ }^{16,18}$ the behavior of these radicals in the glassy state are different 
from that of the solid state in the following two respects: 1. The intensity distribution reversibly changed with temperature. 2. The 9line spectrum was observed even in the radical of oligomeric MMA which was soluble in methanol. Therefore, we must first make clear the origin of the 9-line spectrum in this research. Experiments with triphenylmethyl methacrylate (TPMA) having a bulky ester group and deuterated MMA $\left(\beta-d_{2}\right.$ or $d_{3}$ ) were considered to be a means for further elucidation of the origin.

Herein, we shall report our ESR study on the polymerization of MMA and its homologs in glassy aromatic media and discuss the origin of the 9-line spectrum of the PMMA propagating radical.

\section{EXPERIMENTAL}

\section{Materials}

MMA, commercially available, was washed with an aqueous $5-\%$ sodium bisulfate solution and then in an aqueous saturated sodium chloride solution, and this was followed by drying over anhydrous magnesium sulfate and distillation under reduced pressure. MMA- $\beta-d_{2}$ was prepared by the reaction of methyl hydrogen methylmalonate with formaldehyde- $d_{2}$ in the presence of diethylamine (yield 60\%). ${ }^{14}$ The isotope purity of the monomer was determined to be 98.4\% from mass spectrometry. MMA- $d_{3}$ was similarly prepared from formaldehyde and methyl hydrogen methyl- $d_{3}$-malonate obtained by the reaction of dimethyl malonate with methyl- $d_{3}$ bromide in the presence of sodium methoxide..$^{31,32}$ The isotope purity of the monomer was determined to be $99.9 \%$ from mass spectrometry. The NMR spectra of the deuterated monomers showed that these contained no other isomers. TPMA was kindly provided by Prof. Yuki of the Faculty of Engineering Science, Osaka University. Benzoyl peroxide (BPO), commercially available, was purified by precipitation from its benzene solution with methanol. Anisole and benzonitrile were purified by the methods described in "Organic Solvents.",33 Methyltetrahydrofuran (MeTHF) was refluxed with $\mathrm{LiAlH}_{4}$, distilled over a Vigreux column, dried over sodium wire and distilled again. 2,2,6,6-Tetramethylpiperidinoxyl (Tempo), commercially a- vailable, was used without further purification.

\section{ESR Measurement}

The ESR measurements were made using a Japan Electron Optics Model JES-FE3X ESR spectrometer with $100 \mathrm{kc} / \mathrm{sec}$ modulations. A $\mathrm{TE}_{011}$ mode cylindrical universal cavity with a variable temperature adaptor (ES-UCT-2AX) and UV irradiation window were used. The temperature was controlled by a variation temperature control unit (ES-VT-3A). The spectra were recorded as a first derivative or second derivative of the absorption band. Specimens for these ESR studies were prepared according to the following procedure: $0.75 \mathrm{~m} l$ of a solution of monomer $(4 \mathrm{~mol} / l)$ and BPO $(0.04 \mathrm{~mol} / \mathrm{l})$ in aromatic solvents were placed in a spectrosil sample tube $(5-\mathrm{mm}$ diameter) and degassed by repeated melting and freezing under vacuum. The degassed samples were then sealed under vacuum. The samples were irradiated through the slotted opening of the cavity with light of a 500-W high-pressure mercury arc (ES-UVO5H). A filter (Toshiba filter series UV D1C) was used for isolating $3650 \AA$.

\section{RESULTS}

The change in the spectrum of the polymerization system of MMA with the irradiation time at $-120^{\circ} \mathrm{C}$ is shown in Figure 1. Immediately after the irradiation, the spectrum overlapped with the signal of the radical obtained from the photolysis of BPO in anisole or benzonitrile at $-120^{\circ} \mathrm{C}$. The spectrum changed with the irradiation time, and after nine minutes, a well-resolved spectrum consisting of nine lines with an equal spacing of about $11.5 \mathrm{G}$ and a $g$ value close to that of free spin was obtained. The intensity gradually increased during an irradiation period of $1 \mathrm{hr}$, but the intensity distribution did not change with the time. In addition, no ESR spectrum could be observed in the absence of BPO. These results show that the radical obtained from BPO can add to MMA even in aromatic rigid glass to yield the propagating radical of MMA. A similar spectrum was also recorded at $-160^{\circ} \mathrm{C}$, indicating that the solid-state polymerization could occur even at $-160^{\circ} \mathrm{C}$. Since the polymers obtained in the glassy state were soluble in methanol, the 9-line 


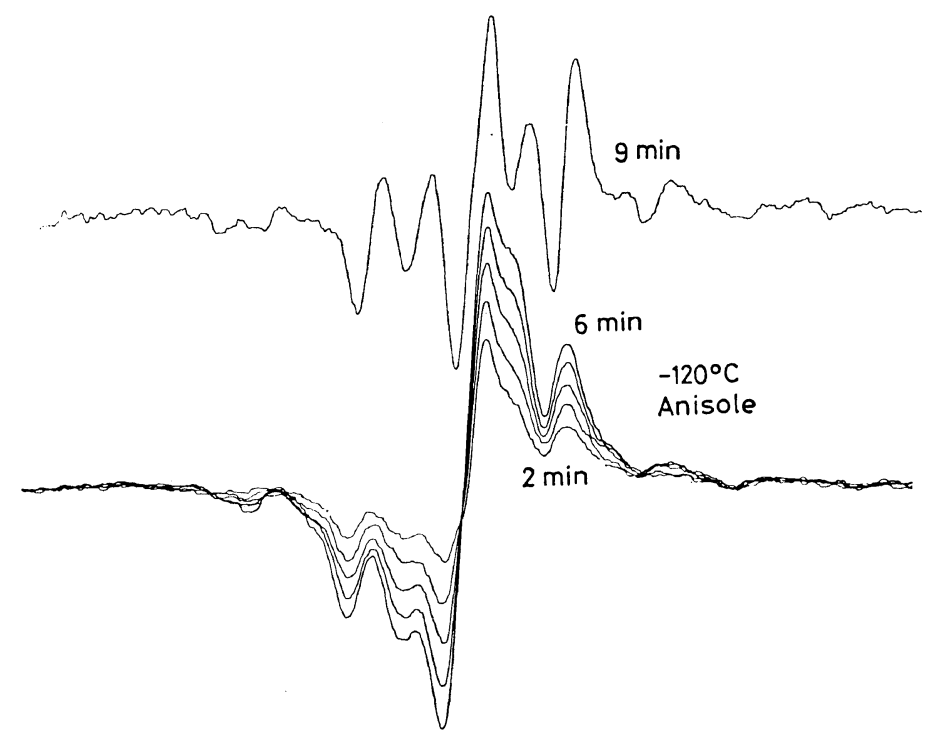

$3 1 \longdiv { 6 3 }$

3263

3363

Figure 1. Change of ESR spectrum with irradiation time in BPO photosensitized polymerization of $\mathrm{MMA}$ at $-120^{\circ} \mathrm{C}$.

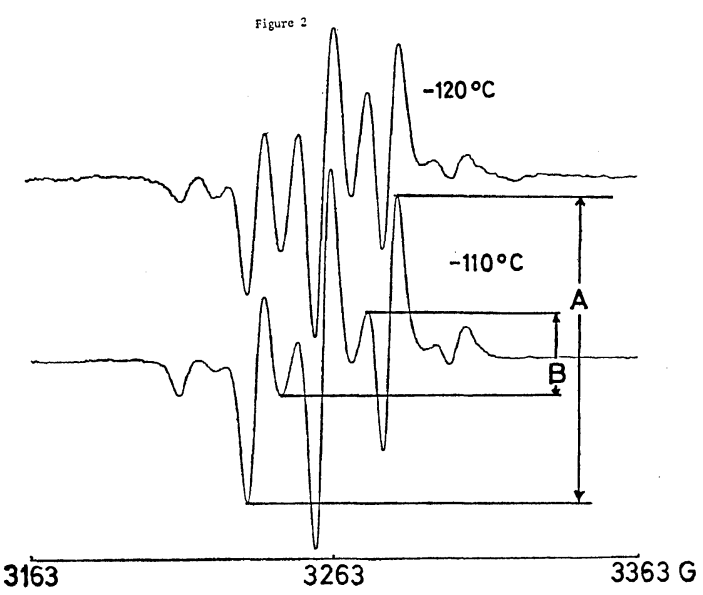

Figure 2. Temperature dependence of the intensity distribution of a PMMA radical in anisole.

spectra could be observed in oligomeric PMMA radical. The intensity distribution of the spectrum changed according to the solvent and the polymerization temperature, as shown in Figures 2 and 3.

For the purpose of estimating the temperature dependence of the relative intensity of the spectrum, a parameter $(R=A / B)$ was defined designating $A$ as the peak-to-peak height of two of stronger five lines and $B$ that of two of weaker four lines. (Figure 2) The $R$ value was independent of the irradiation time at constant temperature after the well-resolved 9-line spectrum appeared. The $R$ value reversibly changed with temperature as shown in Table I.

The variation of the $R$ value with temperature may be correlated with the property of aromatic rigid glass. In order to obtain information for

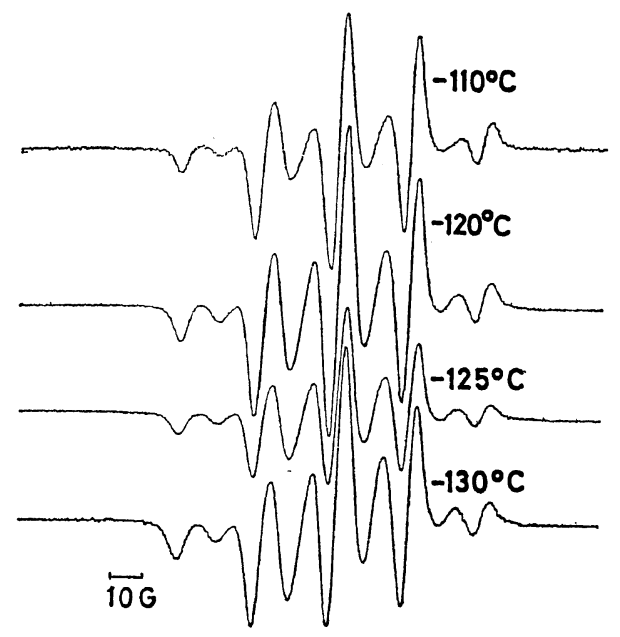

Figure 3. Temperature dependence of the intensity distribution of a PMMA radical in benzonitrile. 
Table I. Solvent and temperature effects on $R$ value ${ }^{a}$

\begin{tabular}{ccc}
\hline \multirow{2}{*}{ Temp, ${ }^{\circ} \mathrm{C}$} & \multicolumn{2}{c}{$R$} \\
\cline { 2 - 3 } & Benzonitrile & Anisole \\
\hline-108 & 3.27 & \\
-110 & 3.11 & 3.48 \\
-115 & 2.64 & 2.51 \\
-120 & 1.98 & 1.58 \\
-125 & 1.63 & \\
-130 & 1.53 & \\
\hline
\end{tabular}

a $R=$ (intensity of signal $\mathrm{A}) /($ intensity of signal $\mathrm{B}$ )

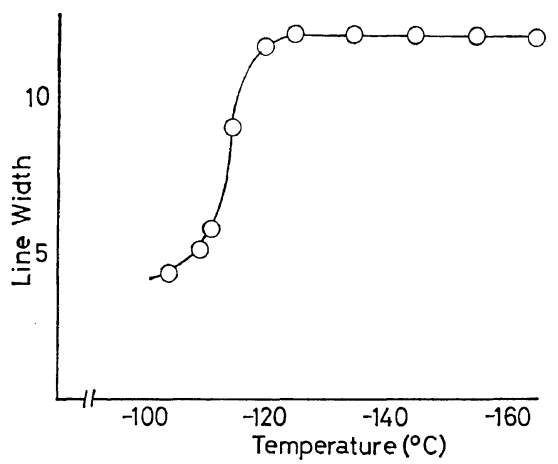

Figure 4. Variation of the line width of Tempo with temperature. MMA-anisole (1:1) mixture.

the thermal behavior of the system, the temperature dependence of the line width of the central line in the ESR spectrum of Tempo was investigated for a 1:1 mixture of MMA and anisole. The result is shown in Figure 4, and suggests the absence of any transition in the interval from $-115^{\circ} \mathrm{C}$ to $-160^{\circ} \mathrm{C}$ and that consequently the change of the spectrum of the propagating radical in this temperature region cannot be ascribed to that of the medium.

The ESR spectra obtained by the polymerization of MMA- $\beta-d_{2}$ and MMA- $d_{3}$ are shown in Figures 5 and 6 , respectively. The former spectrum has four lines $(1: 3: 3: 1)$ with an equal spacing of about $23 \mathrm{G}$, indicating that the methyl group rotates freely and its three protons are equivalent. The intensity of the spectrum did not change below $-120^{\circ} \mathrm{C}$ for $30 \mathrm{~min}$ after interruption of the irradiation. The latter spectrum shows three lines with en equal spacing of about 11.5G. Since the spectrum obtained from MMA- $d_{3}$ is not so well-resolved, the tempera- ture dependence of the spectrum was shown as the second derivative of the absorption spectrum (Figure 7). The relative intensity of the central line increases with a lowering of temperature.

The nonequivalence of $\beta$-protons is considered to increase with the bulkiness of the alkyl group. TPMA which yields stereoregular polymers even at room temperature ${ }^{34,35}$ was studied and the ESR spectrum is shown in Figure 8. The 5-line spectrum $(1: 4: 6: 4: 1)$ with an equal spacing of about 23G was observed for PTPMA, and resonated at the same magnetic fields as those of the five stronger lines of the PMMA radical. The intensity distribution, the line width and the spacing of the line hardly underwent any change with temperature. In addition, the line width of the PTPMA radical is smaller than that of the PMMA radical, indicating that the conformation of the former radical is more limited than that of the latter and is not influenced by change in temperature. The ESR spectrum of the PMMA radical in MeTHF is shown in Figure 9. A 13-line spectrum was

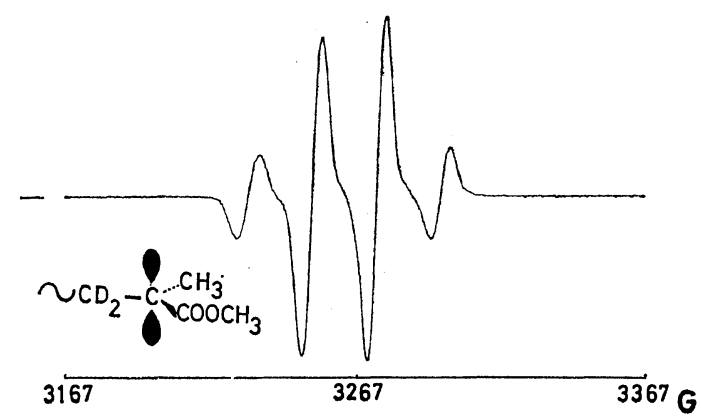

Figure 5. ESR spectrum of PMMA- $\beta-d_{2}$ radical in benzonitrile at $-120^{\circ} \mathrm{C}$.

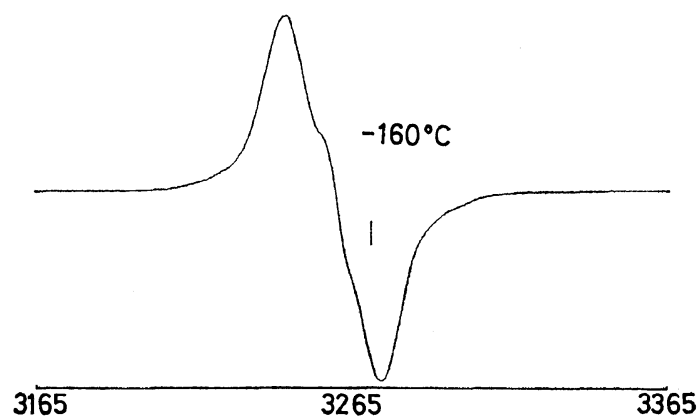

Figure 6. ESR spectrum of PMMA- $d_{3}$ radical in anisole at $-160^{\circ} \mathrm{C}$. 
observed instead of the 9-line spectrum. This spectrum was similar to that reported for the solid-state polymerization of MA, ${ }^{10,16-19,24,28}$ but

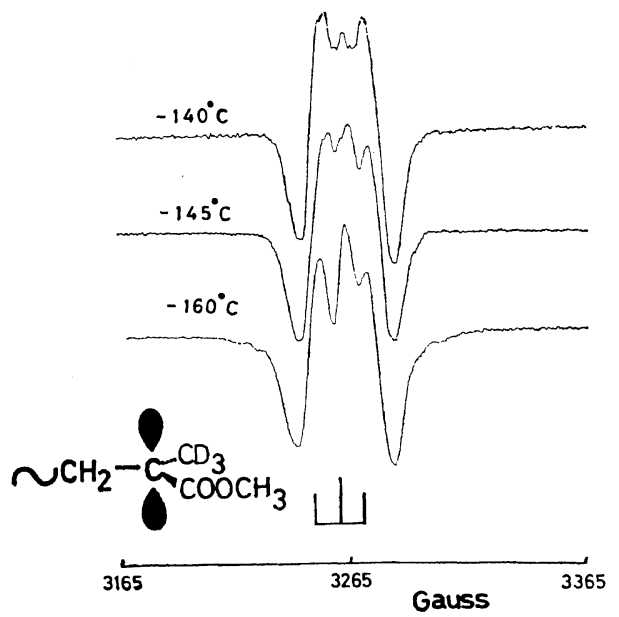

Figure 7. Variation of the ESR spectrum (second derivative) of PMMA- $d_{3}$ radical with polymerization temperature.

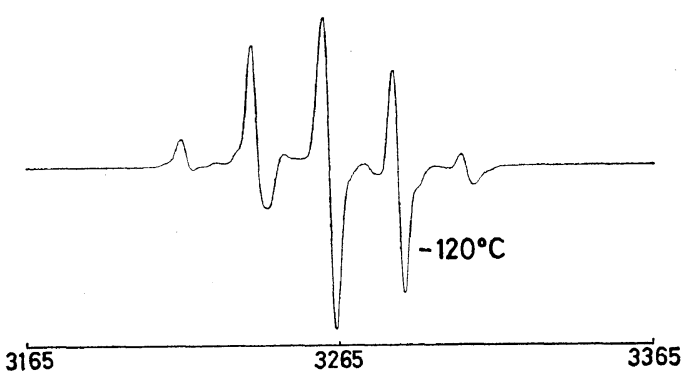

Figure 8. ESR spectrum of PTPMA radical at $-120^{\circ} \mathrm{C}$ in anisole.
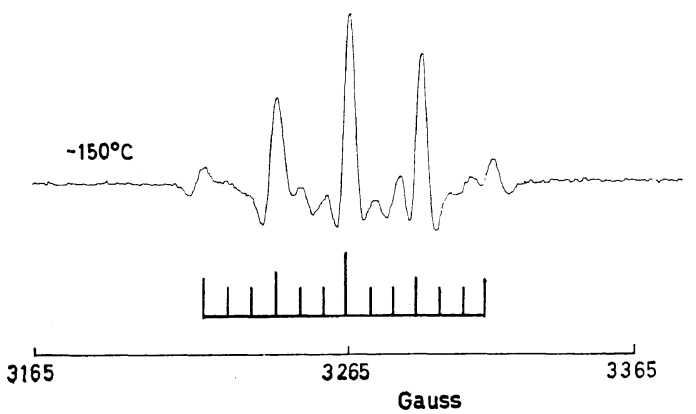

Figure 9. ESR spectrum of PMMA radical in MeTHF at $-150^{\circ} \mathrm{C}$. it did not have the intensity distribution of $1: 1: 1: 4: 3: 3: 6: 3: 3: 4: 1: 1: 1$.

\section{DISCUSSION}

The ESR spectrum of the propagating radical of MMA- $\beta-d_{2}$ is composed of four lines, having an intensity distribution of $1: 3: 3: 1$ and is independent of the temperature, indicating that the $\alpha$-methyl group can freely rotate in the frozen state. The intensity of the 4-line spectrum in MMA- $\beta-d_{2}$ as well as that of the 9-line spectrum in MMA did not change at $-120^{\circ} \mathrm{C}$ for at least $30 \mathrm{~min}$ following interruption of the irradiation. The intensity decreased with time at higher temperatures but the intensity distribution underwent no change until its disappearance. These results suggest that the propagating radical could be composed possibly of a single radical.

The propagating radical obtained from the MMA- $d_{3}$ has a 3-line spectrum of which the intensity distribution depends on the polymerization temperature and which has a hyperfine splitting of about $11.5 \mathrm{G}$. Let us discuss the interpretation of the spectrum in terms of two conformations, ${ }^{6,9,13,25,26,30}$ a Gaussian distribution around the most probable conformation ${ }^{16,18}$ or the exchange of two $\beta$-protons due to the hindered oscillation around the $\mathrm{C}^{\alpha}-\mathrm{C}^{\beta}$ bond of the radical $\left(-\mathrm{C}^{\beta} \mathrm{H}_{2} \dot{\mathrm{C}}^{\alpha}\left(\mathrm{CD}_{3}\right) \mathrm{COOCH}_{3}\right){ }^{18}$

In the case of the two conformations, the 9line spectrum of PMMA radical has been assigned either by the overlap of a 5 -line spectrum $(1: 4: 6: 4: 1)$ and a 4-line spectrum $(1: 3: 3:$ 1), ${ }^{6,25,26,29}$ or that of a 9-line spectrum $(1: 2$ : $4: 6: 6: 6: 4: 2: 1)$ and a 5 -line spectrum $(1: 4:$ $6: 4: 1)^{9,13}$ The former assignment is based on the binomial distribution of the 5-line and 4line spectra. The appearance of the 13-line spectrum in MeTHF is not consistent with this interpretation. The latter assignment is based on $A_{\beta \mathrm{H}}=B \cos ^{2} \theta,{ }^{36}$ where $A_{\beta \mathrm{H}}$ is hyperfine splitting constant to $\beta$-proton, $B$ is $46 \mathrm{G}$ and $\theta$ is dihedral angle shown in Figure 10. Therefore, determination of the angle from the splitting constants observed leads to a symmetrical conformation of the $\beta$-methylene protons around the $C^{\alpha}-C^{\beta}$ bond $\left(\theta_{1}=\theta_{2}=60^{\circ}\right.$, Figure 10) and the 5 -line spectrum becomes a deformed conforma- 

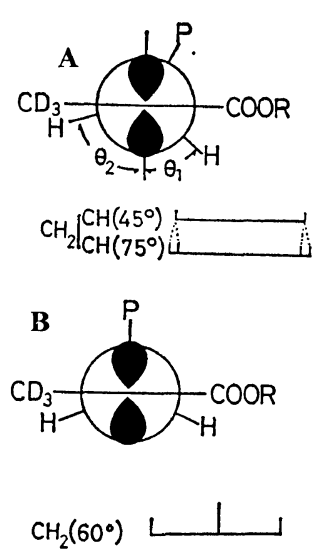

Figure 10. Conformations of PMMA- $d_{3}$ radical and their hyperfine structures in ESR spectrum: (A) deformed conformation; (B) symmetrical conformation.

tion, $\left(\theta_{1}=45^{\circ}\right.$ and $\left.\theta_{2}=75^{\circ}\right) .^{10,17}$ Moreover, the 13-line spectrum of the PMMA radical in MeTHF can be reasonably interpreted as a consequence of a slight distortion of the symmetrical conformation, since a distortion of $5^{\circ}$ around the $C^{\alpha}-C^{\beta}$ bond leads to the 13 lines. ${ }^{10,17}$ The intensity distribution of the spectrum suggests that the 13-line spectrum is composed of two deformed conformations of a single radical.

In the next step, we will discuss the 3-line spectrum of the PMMA- $d_{3}$ radical in terms of the latter interpretation. The symmetrical conformation of the methylene protons results in a 3-line spectrum with a spliting constant of about 11.5G and the deformed conformation in a 2line spectrum with a spliting constant of about 23G. Since the intensity distribution of the 3line spectrum is not binomial, a deformed conformation must be considered in addition to a symmetrical conformation. The reversible change of the intensity distribution with temperature can be ascribed to that of the relative weight of two conformations ( $A$ and $B$ ) coexisting at thermal equlibrium, and the increase in the intensity of the central line of the 3-line spectrum with a lowering of the temperature is compatible with variation in the $R$ value.

The origin of the 3-line spectrum might also be explained as the exchange of two unequivalent $\beta$-protons due to hindered oscillation. ${ }^{19}$ Since the abnormal change of the line width in ESR spectrum of Tempo was not observed from $-115^{\circ} \mathrm{C}$ to $-160^{\circ} \mathrm{C}$, the change in the property of glassy aromatic solvents, such as transition, need not be taken into account in the temperature region. Therefore, if an exchange of $\beta$ protons can occur, the intensity of the central line of the 3-line spectrum is expected to increase with a raise in temperature. But this is not the case, however, an attempt to explain the 9-line spectrum of the PMMA radical has also been made by introducing the concept of the Gaussian distribution of the conformation angle around the most stable conformation. ${ }^{16,18}$ Since there is no abnormal change in the properties of the media in the temperature region measured, the distribution around the most probable conformation would become wide at elevated temperatures. Therefore, the spectra reproduced, assuming the distribution must lead to an increase in the $R$ value by raising the temperature. ${ }^{16,18}$ This expectation is consistent with the temperature dependence on the $R$ value. If this concept is used to explain the ESR spectra of the PTPMA radical, the disappearance of the alternate four lines in the spectra will lead to the view that the distribution of conformation of PTPMA radical angle is broader than that of the PMMA radical. ${ }^{16,18}$ However, these conclusions are completely out of the question for the following reasons: (1) The line width of five lines of PTPMA radical is smaller than that of PMMA radical. (2) The temperature dependence of the ESR spectra of PTPMA radical could hardly find in the temperature region from $-100^{\circ} \mathrm{C}$ to $-150^{\circ} \mathrm{C}$. (3) The Stuart-model inspection showed that the PTPMA radical is quite rigid and cannot undergo further movement. (4) The polymer obtained from the radical polymerization of TPMA is stereoregular even at a polymerization temperature of $60^{\circ} \mathrm{C} .{ }^{35}$ These results suggest that the PTPMA radical has more limited conformations than the PMMA radical. If the concept of two conformations is used to explain the ESR spectra, the 5-line spectra of PTPMA radical indicate that this radical consists of mainly a deformed conformation. This is quite reasonable in consideration of the bulkiness of the ester group.

Accordingly, it is concluded from this ESR study of the photopolymerization of MMA and 
its homolog in the frozen state that the 9-line spectrum of the PMMA radical obtained in the state can be most reasonably explained as two conformations coexisting at a thermal equilibrium.

Acknowledgment. The authors wish to express their sincere appreciation to Professors S. Nozakura and $\mathrm{K}$. Kuwata for their comments and encouragement. They also are indebted to Dr. M. Iwasaki at the Government Industrial Research Institute of Nagoya, Dr. K. Tsuji, Institute for Biological Science of the Sumitomo Chemical Co., and to Dr. Y. Kurita, Basic Research Institute of Toray Co., Prof. H. Yuki and Dr. K. Hatada at Faculty of Engineering Science, Osaka University, do we extend our heart-felt thanks for their providing us with TPMA.

\section{REFERENCES}

1. M. Kamachi, J. Satoh, D. J. Liaw, and S. Nozakura, Macromolecules, 10, 501 (1977).

2. M. Kamachi, D. J. Liaw, and S. Nozakura, Polym. J., 9, 307 (1977).

3. M. Kamachi, J. Satoh, and S. Nozakura, $J$. Polym. Sci. Polym. Chem. Ed., in press.

4. R. Foster, "Organic Charge-Transfer Complex," Academic Press., New York, N.Y., 1969.

5. D. J. E. Ingram, M. C. R. Symons, and M. G. Townsend, Trans. Faraday Soc., 54, 409 (1958).

6. R. J. Abraham, H. W. Meville, D. W. Ovenall, and D. H. Whiffen, ibid., 54, 1133 (1958).

7. D. W. Ovenall, Nature, 184, 181 (1959).

\&. L. H. Piette, "NMR and ESR Spectroscopy," Pergamon Press, Oxford, 1960, p 218.

9. M. C. Symmons, J. Chern. Soc., 1186 (1963).

10. C. H. Bamford, G. C. Eastmond, and Y. Sakai, Nature, 200, 1284 (1963).

11. A. T. Bullock and L. H. Sutcliffe, Trans. Faraday Soc., 60, 625 (1964).

12. J. H. O'Donnell, B. McGarvey, and H. Morawetz, J. Am. Chem. Soc., 86, 2322 (1964).

13. J. Sohma, T. Komatsu, and H. Kashiwabara, J. Polym. Sci., Part B, 3, 287 (1965).

14. P. Kourim and K. Vacek, Trans. Faraday Soc., 61, 415 (1965).
15. R. E. Michel, F. W. Chapman, and T. J. Mao, J. Polym. Sci., Part A-1, 5, 677 (1967).

16. M. J. Bowden and J. H. O'Donnell, J. Phys. Chem., 72, 1577 (1968).

17. C. H. Bamford, A. Bibby, and G. C. Eastmond, Polymer, 9, 629 (1968).

18. M. Iwasaki and Y. Sakai, J. Polym. Sci., Part $A-1,7,1537$ (1969).

19. Y. Sakai and M. Iwasaki, ibid., 7, 1747 (1969).

20. Y. Sakai, ibid., 7, 3177, 3191 (1969).

21. F. Szocs, J. Appl. Polym. Sci., 14, 2629 (1970).

22. T. Gilbro, P. O. Kinell, and A. Lund, J. Polym. Sci., Part A-2, 9, 1495 (1971).

23. M. Iwasaki, K. Nunome, H. Muto, and K. Toriyama, J. Chem. Phys., 54, 1839 (1971).

24. C. Chachaty and A. Forchioni, J. Polym. Sci., Part A-1, 10, 1905, 1923 (1972).

25. S. E. Bresler, E. N. Kozbekov, V. N. Fomichev, and V. N. Shadrin, Makromol. Chem., 157, 167 (1972).

26. J. A. Harris, O. Hinojosa, and J.C. Arthur, Jr., J. Polym. Sci. Polym. Chem. Ed., 11, 3215 (1973).

27. S. E. Bresler, E. N. Kozbekov, V. N. Fomichev, and V. N. Shadrin, Makromol. Chem., 175, 2875 (1974).

28. C. Chachaty, M. Latimier, and A. Forchioni, J. Polym. Sci. Polym. Chem. Ed., 13, 189 (1975).

29. S. E. Bresler, E. N. Kazbekov, and V. N. Shadrin, Vysokomol. Soedin., 17, 584 (1975).

30. H. Yoshioka, H. Matsumoto, S. Uno, and F. Higashide, J. Polym. Sci. Polym. Chem. Ed., 14, 1331 (1976).

31. C. Mannich and K. Ritsert, Ber., 57, 1116 (1924).

32. G. Hilgetag and A. Martini, "Preparative Organic Chemistry," John Wiley, New York, N.Y., 1972, p 912.

33. J. A. Riddick and W. B. Bunger, "Organic Solvents," 3rd ed., Wiley-Interscience, New York, N.Y., 1970, p 711, 808.

34. H. Yuki, K. Hatada, K. Ohta, and Y. Okamoto, J. Polym. Sci., Part B, 6, 753 (1968).

35. J. Niezette and V. Resreux, Makromol. Chem., 149, 177 (1971).

36. C. Heller and H. M. McConnell, J. Chem. Phys., 32, 1535 (1960). 